\title{
Desarrollo de un prototipo recuperador de calor residual de lecho empacado de caliza y coque
}

\author{
Development of a prototype residual heat recuperator packed bed of limestone \\ and coke \\ Oscar Castaño Gallego, Carlos Acevedo Álvarez, Efrén Giraldo Toro \\ Maestría en Gestión Energética Industrial, Instituto Tecnológico Metropolitano, Medellín, Colombia \\ Correo-e: oscarpablo2000@hotmail.com
}

\begin{abstract}
Resumen- En el presente trabajo se diseñó un prototipo de recuperador de calor residual de lecho empacado de caliza y coque, el cual simula la captura de los gases de combustión proveniente de la chimenea de un horno vertical para calcinación de la caliza y obtener cal. El aprovechamiento de estos gases tiene por objeto precalentar un nuevo material de caliza y coque. Dichos gases son expulsados usualmente al medio ambiente con temperaturas superiores a $545^{\circ} \mathrm{C}$. El recuperador consiste en dos tubos de acero inoxidable, uno móvil y uno fijo, el tubo móvil es el recuperador como tal, el tubo fijo cumple la función de soporte, EI recuperador (tubo móvil) tiene un diámetro interno de $0.096 \mathrm{~m}$ y una altura de $0.25 \mathrm{~m}$, el gas utilizado es aire representado por las RPM del ventilador. El lecho empacado es caliza y coque en tamaños de 1 y $1.5 \mathrm{~cm}$, la temperatura es la variable de interés, se registró por medio de 4 termocuplas ubicadas a 4 alturas diferentes al interior del recuperador $(1,7,13 \mathrm{y} 19 \mathrm{~cm})$, los datos se ingresaron en las matrices de Excel avanzado 2010 en la función de regresión lineal múltiple. Excel permitió identificar 2 ecuaciones que predicen el comportamiento de la temperatura de la caliza y el coque. Se encontró que la recuperación de calor en la caliza es del $43 \%$ y para el coque es del $53 \%$ en un tiempo de una hora.
\end{abstract}

Palabras clave- Caliza, Calor residual, Coque, recurso energético.

Abstract - In this paper a prototype waste heat boiler packed bed of limestone and coke, which simulates the capture of flue gases from the chimney of a vertical furnace for calcination of limestone and lime gain was designed. The use of these gases is intended to preheat a new limestone and coke material. These gases are usually driven environment with temperatures above $545^{\circ} \mathrm{C}$. The recovery consists of two stainless steel tubes, one fixed and one mobile, the mobile is the recovery tube as such, the fixed tube serves to support the recovery (mobile tube) having an inner diameter of $0.096 \mathrm{~m}$ and a height of $0.25 \mathrm{~m}$, the gas used is air represented by the fan RPM. The packed bed is limestone and coke in sizes of 1 and $1.5 \mathrm{~cm}$, the temperature is the variable of interest was recorded using 4 thermocouples located at 4 different heights inside the boiler $(1,7,13$ and $19 \mathrm{~cm})$ Data were entered into arrays in Excel 2010 Advanced Function of multiple linear regression. Excel identified two equations predicting the behavior of the temperature of the limestone and coke. It was found that the heat recovery is $43 \%$ limestone and coke is $53 \%$ in a time of one hour.

Key Word - Coke, energy resource, limestone, Waste heat.

\section{INTRODUCCIÓN}

Las actuales tendencias de producción proponen la implementación de tecnologías cada vez más eficientes y amables con el medio ambiente, esto supone un conocimiento profundo de los principios del Uso Racional de la Energía (URE) y de los métodos de diseño termodinámico vía minimización de la tasa de generación de entropía. El sector industrial en Colombia debe apelar a tecnologías ecoeficientes, a fin de disminuir costos por consumo ineficiente de energía y reducir el grado de contaminación que pudiera resultar de los procesos de producción, para posicionarse en el mercado nacional y ampliar sus expectativas de participación competitiva en el mercado internacional.

Muchos procesos industriales generan gran cantidad de calores residuales provenientes de gases de combustión, los cuales son lanzados a la atmósfera desperdiciando un valioso recurso energético, produciendo contaminación térmica, material particulado y aumentando gases contribuyentes al calentamiento global.

Cualquier corriente de gas residual con temperaturas superiores a $121^{\circ} \mathrm{C}$ [1] son susceptibles de recuperación, el calor residual puede ser utilizado en varios procesos de precalentamiento: carga en un horno, aire de combustión, de agua, de edificios, entre otros. Para la producción de cal, la materia prima principal es la caliza, inicia el calentamiento a $25^{\circ} \mathrm{C}$ en la etapa de puesta en marcha en el horno vertical, la calcinación de la caliza se 
logra a $900^{\circ} \mathrm{C}$, luego los gases de combustión salen a través de la parte superior del horno a temperaturas superiores de $545^{\circ} \mathrm{C}$, este gas residual se puede redireccionar mediante recuperadores de calor para el precalentamiento de un nuevo material de caliza y coque, este precalentamiento puede significar un importante ahorro de combustible y costos generales del proceso, adicional a ello la industria de la cal puede revender créditos de carbono por dicha recuperación (Bolsa Climática de Chicago), reduciendo la contaminación térmica y material particulado, ya que el recuperador puede actuar como filtro y aprovechar el calor residual mejorando la eficiencia térmica del proceso [2].

En la región antioqueña y especialmente en la región oriental (Magdalena central), se encuentran varias empresas dedicadas a la producción de cal y cemento, debido a la gran cantidad de yacimientos de caliza en la región, muchos de los sistemas energéticos utilizados por estas empresas son obsoletos e ineficientes. Los recuperadores de calor de lecho empacado de caliza y carbón son una buena opción debido a su bajo costo, fácil construcción y reducción en los tiempos de operación, pero aún falta optimizar los recuperadores de calor de lecho empacados, en especial los de caliza, se deben optimizar las condiciones de operación, los perfiles de temperatura y almacenamiento de calor, y el efecto en el tipo de geometría.

Los efectos de las anteriores variables no se han estudiado con propiedad en el precalentamiento de la caliza [2]. Por lo anterior, se hace apropiado realizar esta investigación a nivel de tesis de Maestría en Gestión Energética Industrial sobre recuperadores de calor de lecho empacado de caliza y coque.

Los antecedentes de esta investigación se registran en la década de los 90's en la Universidad de Antioquia, donde se diseñó y construyó un sistema de recuperación de calor residual para los gases de escape del horno de cubilote del taller de fundición [3]. Se diseñaron dos regeneradores térmicos de lecho empacado de esferas metálicas, se recuperaron calores con temperaturas de $450{ }^{\circ} \mathrm{C}$, cumpliendo las normas para el medio ambiente. Se logró un aumento de 50\% en su producción, elevó la temperatura del metal en $7 \%$ y disminuyó el contenido de azufre en $60 \%$ respecto a la operación con soplo frío [4].

En 1999 se inició el proyecto Optimización de la Planta Piloto Eco-eficiente de la Universidad de Antioquia, financiado por el Centro de Ciencia y Tecnología de Antioquia, para ser desarrollado conjuntamente por el Grupo de Investigación en Ingeniería y Ciencias Térmicas (DRAGON), de la Universidad Nacional y el Grupo de Investigaciones Pirometalúrgicas y de Materiales (GIPIMME), de la Universidad de Antioquia. En este proyecto se trataba de mejorar los aspectos termomecánicos del horno de cubilote del taller de fundición, con el fin de reducir costos para ponerlo al alcance de las PYMES del sub-sector Fundición.

La optimización del lecho poroso se planteó en términos de mejorar la relación entre transferencia de calor y pérdida de presión. El proyecto entregó como resultados el planteamiento de un parámetro de optimización [4], las funciones de aproximación a la distribución de temperatura del lecho empacado sometido a convección forzada transitoria [5] y [6], un análisis exergético de los gases de escape de la planta, el rediseño de dos válvulas de cuatro vías y una unión flexible en los ductos del sistema. Al finalizar este proyecto, quedo planteada la necesidad de profundizar en el estudio de la fenomenología termodinámica de los regeneradores térmicos (recuperadores de calor), con el fin de estructurar un procedimiento de diseño y optimización más preciso que los hasta ahora utilizados.

\section{CONTENIDO}

Para el diseño del presente prototipo se construyó un horno o cámara de calentamiento, el cual simula el horno vertical en el proceso real: Consiste en un recinto construido de material aislante (ladrillo aislante UA-23 de la empresa Erecos), con un espesor de pared suficiente para evitar pérdidas de calor por conducción, así como también fugas de aire suministrado por un ventilador instalado en la parte inferior de la cámara, el ventilador proporciona el gas (aire) forzado transitorio, no se utilizan gases de combustión debido a los altos costos para intentar reproducirlo en el laboratorio, igualmente debido a que en el proceso ideal solo se anhela precalentar la caliza y el coque, materiales para obtener cal.

La cámara se construyó con un circuito resistivo de $3600 \mathrm{~W}$ obteniendo un mejor control del proceso. Para sostener la temperatura de $545^{\circ} \mathrm{C}$ al interior de la cámara, se utilizó un termocupla tipo $\mathrm{K}$ conectado a un controlador digital de temperatura (EBCHQ 58008). El gas o aire proveniente del ventilador entra a la cámara a temperatura ambiente, pasa a través del circuito resistivo, se calienta y sale de la cámara a una temperatura de $545^{\circ} \mathrm{C}$, el gas caliente que pasa posteriormente al recuperador con el objetivo de precalentar el material de caliza y coque en su interior.

El recuperador de calor consiste en un sistema de dos tubos, el primero es un tubo fijo de acero inoxidable al cromo níquel (188) \%, con una resistencia mecánica de $60 \mathrm{kgf} / \mathrm{mm}^{2}$ y una dureza de $175-200 \mathrm{Hb}$, tiene un diámetro interno de $0.108 \mathrm{~m}$ y una altura de $0.23 \mathrm{~m}$, en él se soldó una arandela del mismo material que sirve como soporte para asegurarlo a la cámara de calentamiento y su función es la de sostener en su interior un tubo móvil (es móvil debido a la facilidad para retirarlo o introducirlo en el tubo fijo y poder llenarlo o vaciarlo con un nuevo material para una nueva prueba). El recuperador como tal consiste en un tubo de acero inoxidable al cromo níquel (188) \% de menor diámetro, en la parte inferior se soldó una rejilla de acero inoxidable para que retenga el material a precalentar pero que igual permita el ingreso del gas caliente, el tubo móvil tiene un diámetro interno de $0.096 \mathrm{~m}$ y una altura de $0.25 \mathrm{~m}$. Ver figura 1. 


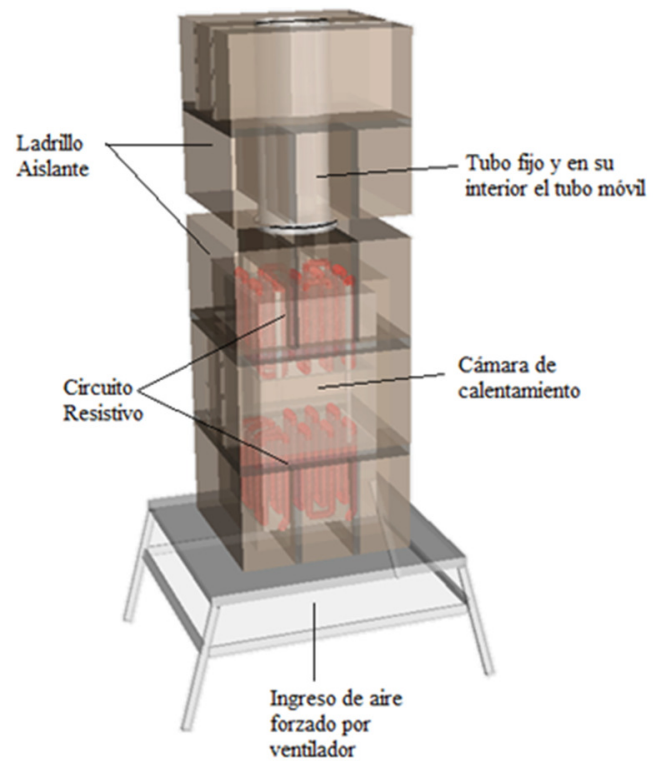

Figura 1. Conjunto de Cámara de calentamiento y recuperador

Una vez ensamblado el conjunto de cámara de calentamiento y el recuperador, se procedió a instalar los equipos de medición restantes. Se instalan 4 termocuplas al interior del recuperador a diferentes alturas $(0.19,0.13,0.07$ y $0.01 \mathrm{~m})$, se conectan a igual número de medidores digitales de temperatura (EBCHQ 58008), los que registrarán el progreso de la temperatura al interior del recuperador. Ver figura 2.

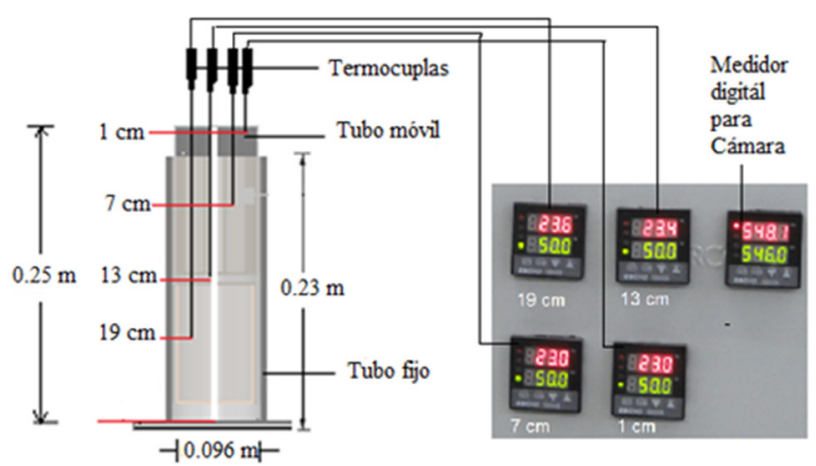

Figura 2. Sistema de medidores digitales conectados a las termocuplas al interior del recuperador (tubo fijo y móvil)

A la cámara de calentamiento en su parte inferior se realizó un orificio a través de la cual se instaló un sistema de tuberías para conducir el aire proveniente del ventilador. En el ventilador se instaló un variador de velocidad (marca Siemens GSE6440), se halló la frecuencia del ventilador y se determinó las RPM del ventilador. La figura 3 muestra el sistema completo.

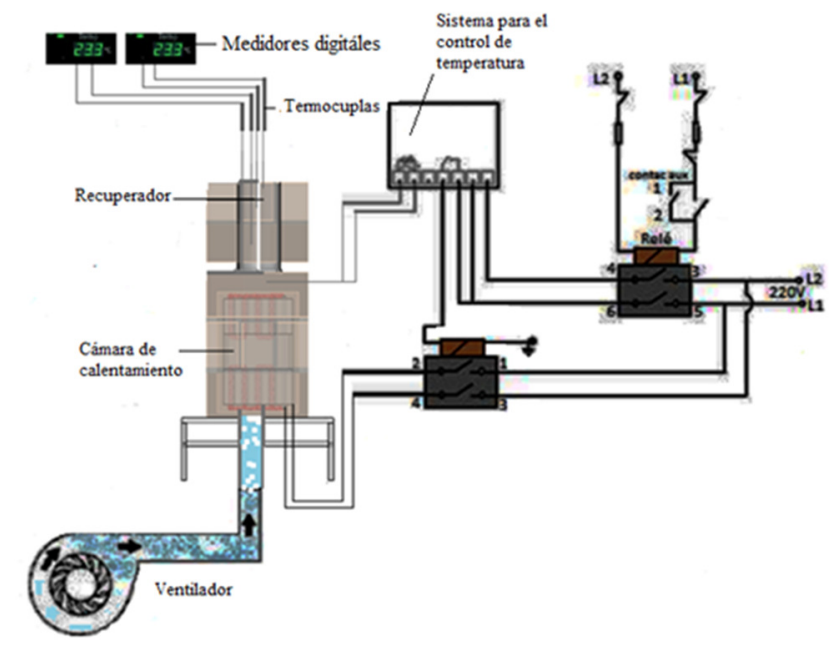

Figura 3. Sistema completo de cámara, recuperador y sistemas de medición.

\section{PROCEDIMIENTO}

Para el diseño experimental se definieron tres tipos de variables: fijas, de incidencia y las variables de respuesta. Para las variables fijas se tienen: el diámetro interior del recuperador (tubo móvil, $\Phi=0.096 \mathrm{~m})$, la altura del recuperador $(0.25 \mathrm{~m})$, el volumen $(\mathrm{V})$ del recuperador es:

$$
V=\pi\left(\frac{0.096}{2}\right)^{2} * 0.25=1.8095 * 10^{-3} \mathrm{~m}^{3}
$$

El voltaje para la cámara de calentamiento $(220 \mathrm{~V})$, intensidad eléctrica (16,36 A), resistencia fija (13.44 $\Omega$ ) y la potencia de $3600 \mathrm{~W}$. En cuanto a las variables de incidencia se tienen tres: la Velocidad del aire, representada en los RPM (Revoluciones por Minuto) del motor del ventilador, lo que reportó 2 niveles de velocidad; se utilizaron las velocidades recomendadas entre 2.1 y $3.1 \mathrm{~m} / \mathrm{s}$ [7], debido a que estas deben garantizar que el flujo del aire al interior del recuperador sea turbulento (Reynolds > 4000). La segunda variable de incidencia fue el Tamaño de partícula con dos niveles de 0.01 y $0.015 \mathrm{~m}$ de diámetro, esto permitió determinar una relación de diámetro de tubo/diámetro de partícula (dT/dp) entre 6.4 y 9.6, relación que se encuentra dentro de los parámetros propuestos por Benyahia y Oneill [8]. Para un diámetro de tubo de 0.096 m y relación dT/dp, tenemos la ecuación 2:

$$
\frac{d T}{d p}=\frac{0.096}{0.01}=9.6 \text { y } \frac{0.096}{0.015}=6.4
$$

La última variable de incidencia es la Altura del recuperador, para ello se tomarán medidas de temperatura cada $6 \mathrm{~cm}$, debido a que el espacio para la introducción de las termocuplas es reducido, por lo que solo admite 4 termocuplas al interior del recuperador, esto permitirá determinar la incidencia de la velocidad del aire y el tamaño de partícula en la transferencia de calor. Las variables de respuesta serán el calor recuperado mediante la medición de la temperatura. 
El primer paso para la puesta en marcha fue determinar las revoluciones (RPM) del ventilador, estas se registraron mediante un analizador de frecuencia y se midió la velocidad del aire mediante un anemómetro (Digital Anemometer GM8901-00). Se reportaron los siguientes datos, a $20 \mathrm{~Hz}$ se obtuvo 1194 RPM y una velocidad del aire de $1.1 \mathrm{~m} / \mathrm{s}$, a una frecuencia de $40 \mathrm{~Hz}$ para $2388 \mathrm{RPM}$ y una velocidad de aire de $2.1 \mathrm{~m} / \mathrm{s}$ y una frecuencia de $60 \mathrm{~Hz}$ se obtuvo $3577 \mathrm{RPM}$ y una velocidad del aire de $3.1 \mathrm{~m} / \mathrm{s}$. Las revoluciones predominantes serán las de 2388 y 3577 RPM, debido a que son las más cercanas a las velocidades recomendadas [6], pero igualmente fue utilizada 1194 RPM para analizar el precalentamiento de coque. Los datos son reportados en la tabla 1.

Tabla 1. Datos obtenidos según frecuencia medida en el ventilador.

\begin{tabular}{|c|c|c|c|}
\hline $\begin{array}{l}\text { Frecuencia } \\
(\mathrm{Hz})\end{array}$ & RPM & $\begin{array}{l}\text { Velocidad } \\
\text { del aire } \\
\left(\frac{m}{s}\right)\end{array}$ & $\begin{array}{l}\text { Caudal de } \\
\text { aire }\left(\frac{m^{3}}{s}\right)\end{array}$ \\
\hline 20 & 1194 & 1.1 & 0.0079 \\
\hline 40 & 2388 & 2.1 & 0.0152 \\
\hline 60 & 3577 & 3.1 & 0.0224 \\
\hline
\end{tabular}

\section{RESULTADOS}

Se presentan los resultados de caliza en el recuperador con un tamaño de partícula de $1 \mathrm{~cm}$ y un RPM de 2388. Ver Figura 4.

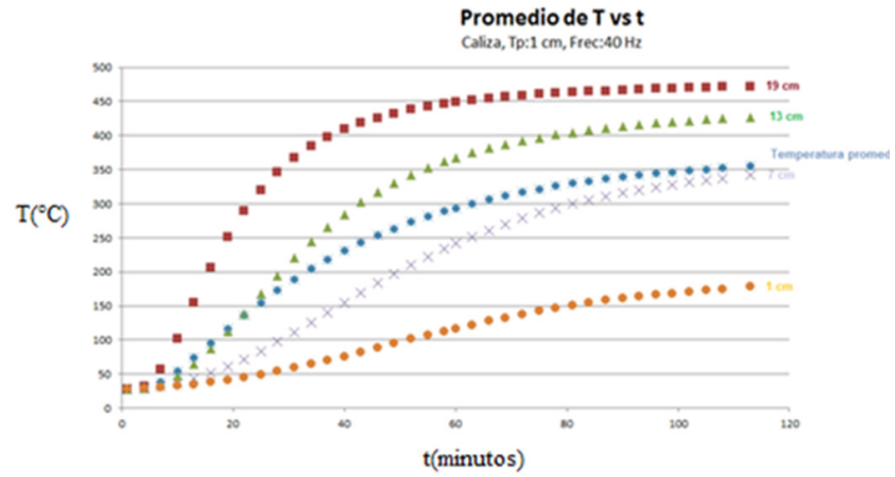

Figura 4. Promedio de temperatura de caliza, Tp: $1 \mathrm{~cm}$ y 2388 RPM.

La figura 4 proporciona datos típicos del precalentamiento de caliza, el tiempo empleado para esta prueba fue de 113 minutos, se pretendía obtener la tendencia del promedio de temperatura a lo largo del tiempo, la termocupla $1(19 \mathrm{~cm})$ obtuvo una 472.4 ${ }^{\circ} \mathrm{C}$, la termocupla $2(13 \mathrm{~cm})$ obtuvo $427.5^{\circ} \mathrm{C}$, la termocupla 3 $(7 \mathrm{~cm})$ obtuvo $341.9{ }^{\circ} \mathrm{C}$ y la termocupla $4(1 \mathrm{~cm})$ obtuvo $178.3^{\circ} \mathrm{C}$; para un promedio de temperatura máxima de $355^{\circ} \mathrm{C}$, con una recuperación de $168.79 \mathrm{kcal}(669.81 \mathrm{BTU})$, cálculo que se realizó con base en la ecuación 3 .

$$
Q=* m * C e * \Delta T
$$

Se obtiene:

$$
Q_{\text {caliza }}=(2.325) *(0.22) *(355-25)=168.79 \mathrm{kcal}
$$

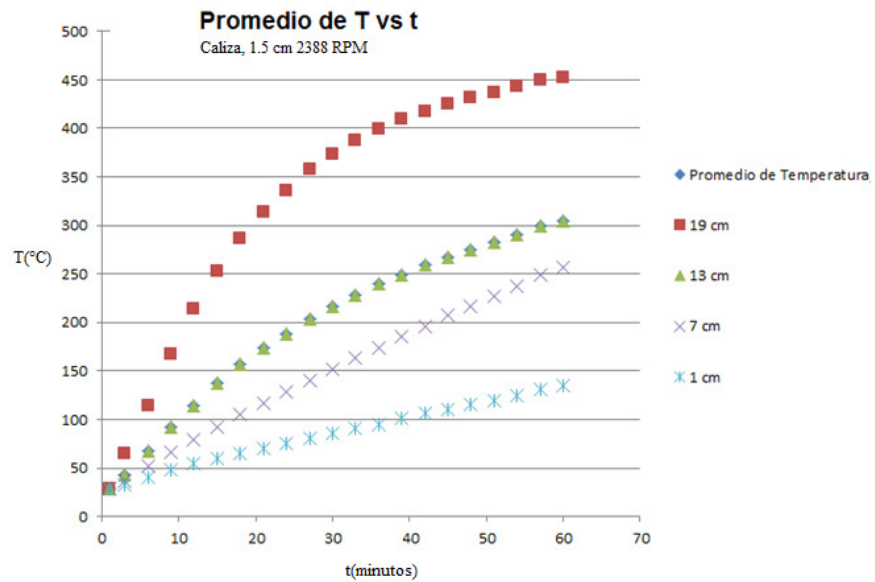

Figura 5. Promedio de temperatura de caliza, Tp: $1.5 \mathrm{~cm}$ y 2388 RPM.

En la figura 5 se analizó el calentamiento de caliza de $1.5 \mathrm{~cm}$ de tamaño de partícula y 2388 RPM en un tiempo de 60 minutos, muestra una tendencia igual al calentamiento realizado con la caliza de $1 \mathrm{~cm}$ en el mismo lapso (60 minutos) con una temperatura de máxima de $452.2{ }^{\circ} \mathrm{C}$ en la termocupla 1, una máxima de $373.5^{\circ} \mathrm{C}$ en la termocupla $2,257^{\circ} \mathrm{C}$ en la termocupla 3 y de $135^{\circ} \mathrm{C}$ en la termocupla 4 , el promedio de temperatura es de $304.4^{\circ} \mathrm{C}$, para un calor recuperado de 142.13 kcal (56.71 BTU), con base en la ecuación 3 , se obtiene:

$$
Q_{\text {caliza }}=(2.325) *(0.22) *(304.4-25)=142.13 \mathrm{kcal}
$$

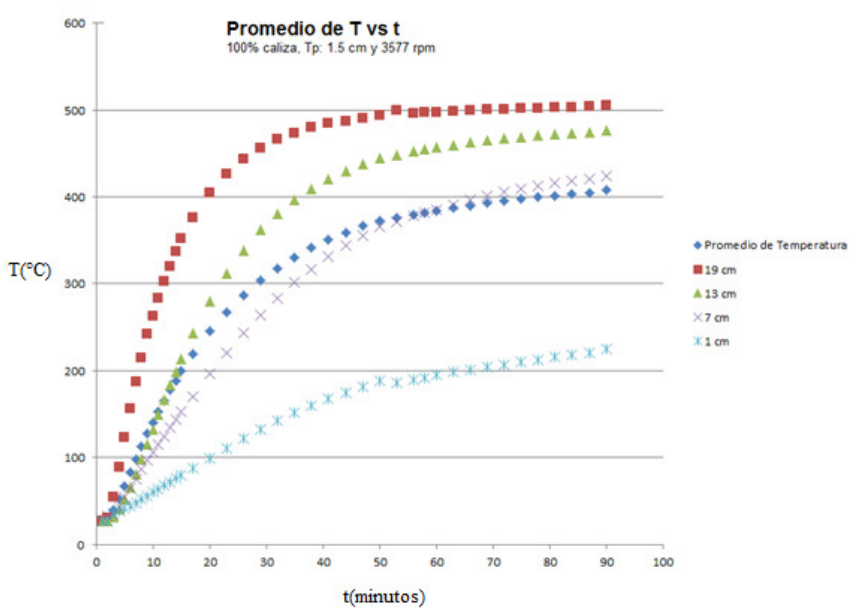

Figura 6. Calentamiento de caliza, Tp: $1.5 \mathrm{~cm}$ y 3577 RPM.

En la figura 6 encontramos un calentamiento de caliza de 1.5 $\mathrm{cm}$ y 3577 RPM, realizado en 90 minutos, en donde la termocupla 1 alcanzó una máxima de $504.8^{\circ} \mathrm{C}$, la termocupla 2 registró $476.2{ }^{\circ} \mathrm{C}$, la termocupla 3 mostró $423.8{ }^{\circ} \mathrm{C}$ y la 
termocupla 4 evidenció $225,5^{\circ} \mathrm{C}$. La temperatura promedio fue de $407.57{ }^{\circ} \mathrm{C}$, para una recuperación de calor de $195.68 \mathrm{kcal}$ (776.52 BTU), con base en la ecuación 3, se obtiene:

$$
Q_{\text {caliza }}=(2.325) *(0.22) *(407.57-25)=195.68 \mathrm{kcal}
$$

Comenzaremos el análisis del coque con un tamaño de partícula de $1 \mathrm{~cm}$ y 2388 RPM, la figura 7 representa lo encontrado en esta prueba.

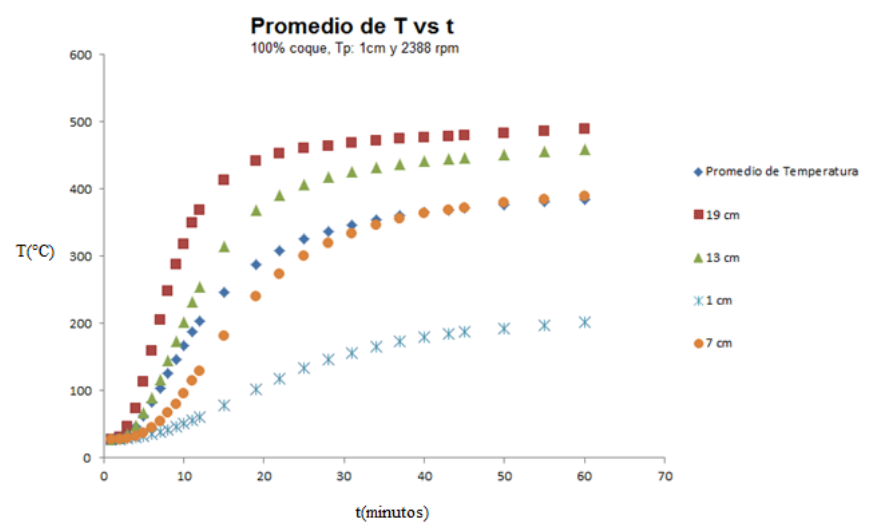

Figura 7. Calentamiento de coque, con Tp: $1 \mathrm{~cm}$ y 2388 RPM.

Para el coque de la figura 7 se realizó un calentamiento en 60 minutos, no se detectó ignición, la termocupla 1 presenta 489 ${ }^{\circ} \mathrm{C}$, la termocupla 2 registra $458.9^{\circ} \mathrm{C}$, la termocupla 3 muestra 390.1 y la termocupla 4 evidencia $201.4^{\circ} \mathrm{C}$. La temperatura promedio alcanzada fue de $384.85^{\circ} \mathrm{C}$ para una recuperación de calor de $48.21 \mathrm{kcal}$ (191.31 BTU), con base en la ecuación 3, se obtiene:

$$
Q_{\text {coque }}=(0.67) *(0.2) *(384.85-25)=48.21 \mathrm{kcal}
$$

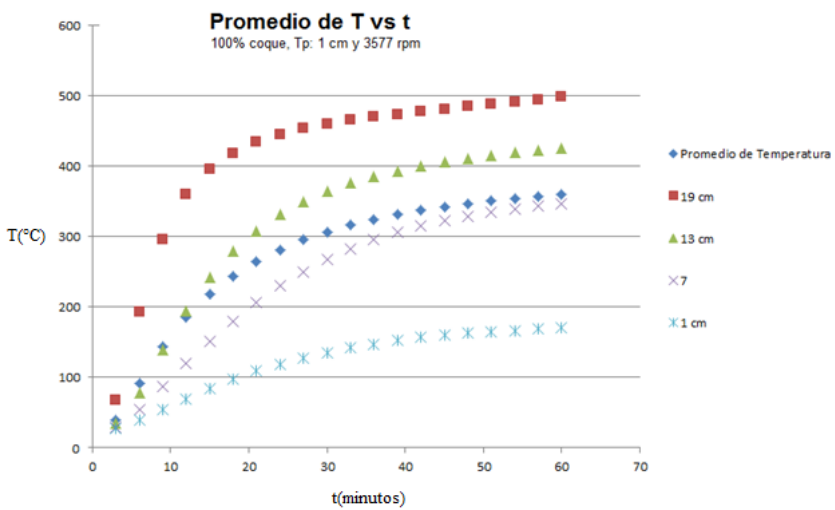

Figura 8. Calentamiento de coque, con Tp: $1 \mathrm{~cm}$ y 3577 RPM.

De la figura 8 con coque de $1 \mathrm{~cm}$ y 3577 RPM, el tiempo de la prueba fue de 60 minutos, no se observó ignición del coque, la termocupla 1 obtuvo una máxima de $498.9{ }^{\circ} \mathrm{C}$, la termocupla 2 registró $426.4^{\circ} \mathrm{C}$, la termocupla 3 mostró 346.7 y la termocupla 4 señaló $170.6{ }^{\circ} \mathrm{C}$; para un promedio de $360.65{ }^{\circ} \mathrm{C}$, para una recuperación de $44.97 \mathrm{kcal}$ (178.45 BTU), con base en la ecuación 3 , se obtiene:

$$
Q_{\text {coque }}=(0.67) *(0.2) *(360.65-25)=44.97 \mathrm{kcal}
$$

Analizamos la figura 9, calentamiento de coque con Tp: 1.5 y 2388 RPM.

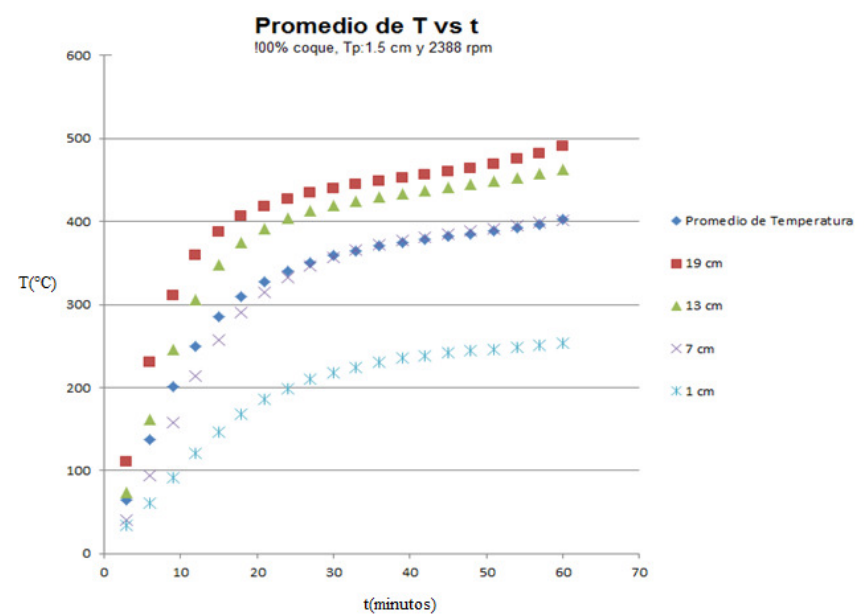

Figura 9. Calentamiento de coque, Tp:1.5 cm y 2388 RPM.

En la figura 9 el coque se calentó durante 60 minutos, se observa un leve cambio en la temperatura de la termocupla 1 cerca de $450{ }^{\circ} \mathrm{C}$, pero no se pude afirmar o negar si existió ignición del coque, debido a que no se superó la temperatura de control $\left(546{ }^{\circ} \mathrm{C}\right)$, registrándose una máxima de $490.7^{\circ} \mathrm{C}$ en la termocupla 1 , la termocupla 2 mostró $463^{\circ} \mathrm{C}$, en la termocupla 3 se evidencia una máxima de $402{ }^{\circ} \mathrm{C}$ y en la cuarta muestra 253.6, el promedio de temperatura fue de 402.32, para una recuperación de calor de $50.56 \mathrm{kcal}$ (200.63 BTU), con base en la ecuación 3 , se obtiene:

$$
Q_{\text {coque }}=(0.67) *(0.2) *(402.32-25)=50.56 \mathrm{kcal}
$$

\section{ANÁLISIS DE RESULTADOS}

Comenzamos el análisis con la caliza en el recuperador. En la tabla 2 se reporta la matriz para caliza. 
Tabla 2. Matriz para ser incorporada a Excel 2010 para caliza.

\begin{tabular}{|c|c|c|c|c|c|c|c|}
\hline $\begin{array}{c}\mathrm{X} 1) \mathrm{T} \\
\text { amaño } \\
\text { de } \\
\text { partíc } \\
\text { ula } \\
\text { (cm) }\end{array}$ & $\begin{array}{c}\mathrm{X} 2) \\
\text { Caud } \\
\text { al } \\
\left(\mathrm{m}^{3} /\right. \\
\mathrm{s})\end{array}$ & $\begin{array}{c}\mathrm{X} \\
3) \\
\% \\
\mathrm{Cal} \\
\text { iza }\end{array}$ & $\begin{array}{l}\mathrm{X} \\
4) \\
\% \\
\mathrm{Co} \\
\mathrm{qu} \\
\mathrm{e}\end{array}$ & $\begin{array}{l}\text { (X5)Coq } \\
\text { ue/caliza }\end{array}$ & $\begin{array}{l}\text { (X6)Cali } \\
\text { za/coque }\end{array}$ & $\begin{array}{l}\text { (X7)Tiemp } \\
\text { o(minutos) }\end{array}$ & $\begin{array}{l}(\mathrm{Y}) \mathrm{Tem} \\
\text { peratura } \\
\left({ }^{\circ} \mathrm{C}\right)\end{array}$ \\
\hline 1 & $\begin{array}{c}0,015 \\
2\end{array}$ & 1 & 0 & 0 & 0 & 113 & 355 \\
\hline 1 & $\begin{array}{c}0,022 \\
4\end{array}$ & 1 & 0 & 0 & 0 & 60 & 299,4 \\
\hline 1,5 & $\begin{array}{c}0,015 \\
2\end{array}$ & 1 & 0 & 0 & 0 & 60 & 304,4 \\
\hline 1,5 & $\begin{array}{c}0,022 \\
4\end{array}$ & 1 & 0 & 0 & 0 & 90 & 407,57 \\
\hline
\end{tabular}

En Excel 2010 se registran los datos hallados y obtenemos los factores de regresión lineal múltiple para los datos obtenidos de Caliza (100\%), reportando lo siguiente:

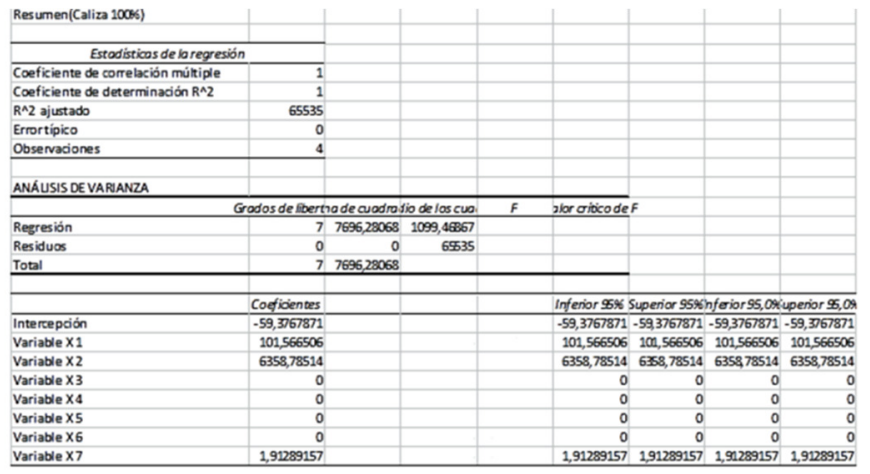

Tenemos un coeficiente de correlación de 1 por lo que la ecuación hallada genera una confiabilidad del 100\%, el efecto constante global es -59.37 , la variable dominante es el caudal de aire, con un factor de X2 $=6358.78$, el segundo factor determinante es el tamaño de partícula $X 1=101.56$ y el factor del tiempo $\mathrm{X} 7=1.912$, las variables $\mathrm{X} 3, \mathrm{X} 4, \mathrm{X} 5$ y X6 no influyen en el proceso. La ecuación de regresión lineal múltiple para caliza $100 \%$ es:

$$
T_{4}=-59.37+101.56 X 1+6358.78 X 2+1.912 X 7
$$

Es decir, si se necesita obtener idealmente una temperatura de $546{ }^{\circ} \mathrm{C}$ en todo el recuperador con un tamaño de partícula de 1 $\mathrm{cm}$ y un caudal de $0.0152 \mathrm{~m}^{3} / \mathrm{s}$, el tiempo requerido será de 212.95 minutos cuando se tiene caliza $100 \%$. Si por ejemplo deseamos obtener $546{ }^{\circ} \mathrm{C}$ para el precalentamiento de caliza de $1.5 \mathrm{~cm}$ y un caudal de $0,0224 \mathrm{~m}^{3} / \mathrm{s}$, el tiempo será de 162.44 minutos.

Si se trata de precalentar el coque, lo recomendable es analizar los datos usando un caudal de $0.0079 \mathrm{~m}^{3} / \mathrm{s}$ (1194 RPM) para los dos tamaños de partícula de interés, ya que la ignición del coque involucra cambios químicos en su composición, los cuales no son uno de los objetivos de la presente investigación. Igualmente importante identificar tiempos diferentes del precalentamiento, para que Excel Avanzado no reporte la variable tiempo como cero, para ello nos remitimos a la figura 9, en la cual la temperatura promedio en un tiempo de 30 minutos es aproximada a $267.65{ }^{\circ} \mathrm{C}$. Datos reportados en la matriz de la tabla 3.

Tabla 3. Matriz para ser ingresada en Excel para coque.

\begin{tabular}{|c|c|c|c|c|c|c|c|}
\hline $\begin{array}{l}(\mathrm{X} 1) \\
\text { Tama } \\
\text { ño de } \\
\text { partíc } \\
\text { ula } \\
(\mathrm{cm})\end{array}$ & $\begin{array}{l}(\mathrm{X} 2 \\
) \\
\text { Cau } \\
\text { dal } \\
( \\
m^{3} / \\
s)\end{array}$ & $\begin{array}{l}\text { (X3 } \\
\text { \% } \\
\text { Cali } \\
\text { za }\end{array}$ & $\begin{array}{l}\text { X4 } \\
) \% \\
\text { Coq } \\
\text { ue }\end{array}$ & $\begin{array}{l}\text { (X5) } \\
\text { Coque/c } \\
\text { aliza }\end{array}$ & $\begin{array}{l}\text { (X6) } \\
\text { Caliza/c } \\
\text { oque }\end{array}$ & $\begin{array}{l}\text { (X7) } \\
\text { Tiempo(minu } \\
\text { tos) }\end{array}$ & $\begin{array}{l}(\mathrm{Y}) \\
\text { Temper } \\
\text { atura } \\
\left({ }^{\circ} \mathrm{C}\right)\end{array}$ \\
\hline 1 & $\begin{array}{l}0.00 \\
79\end{array}$ & 0 & 1 & 0 & 0 & 60 & 302,7 \\
\hline 1.5 & $\begin{array}{l}0.00 \\
79\end{array}$ & 0 & 1 & 0 & 0 & 30 & 267,65 \\
\hline
\end{tabular}

En Excel avanzado para estadística se introducen los datos hallados y obtenemos los factores de regresión lineal múltiple para los datos obtenidos de coque, reportando lo siguiente:

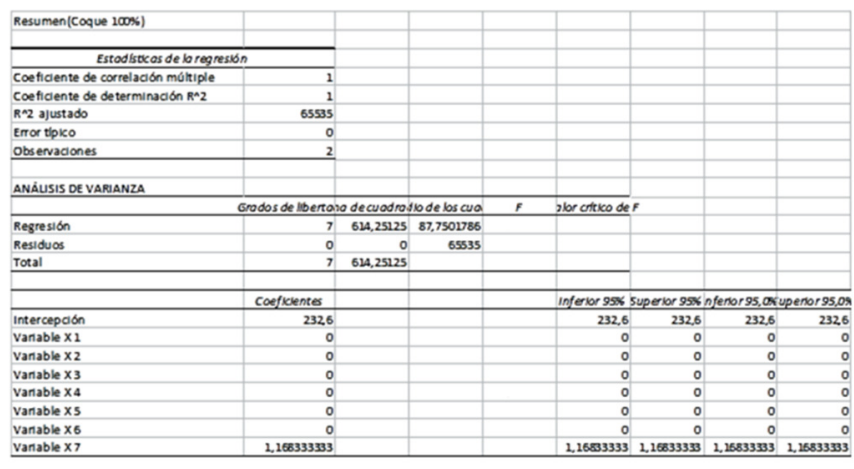

Como el caudal es igual para los dos experimentos, Excel da como respuesta para esta variable cero, tenemos un coeficiente de correlación de 1 por lo que la ecuación hallada genera una confiabilidad del $100 \%$, el efecto constante global es 232.6, la variable dominante es el tiempo, con un factor de $X 7=1.16$, las variables $\mathrm{X} 1, \mathrm{X} 2, \mathrm{X} 3, \mathrm{X} 4, \mathrm{X} 5$ y X6 no influyen en el proceso, la ecuación de regresión lineal múltiple para el coque $100 \%$ es:

$$
T_{3}=232.6+1.168 X 7
$$

Es decir que si necesitamos obtener idealmente una temperatura de $546{ }^{\circ} \mathrm{C}$ en todo el recuperador con coque con un tamaño de partícula de $1 \mathrm{~cm}$ y un caudal de $0.0079 \mathrm{~m}^{3} / \mathrm{s}$, el tiempo requerido será de 268.32 minutos.

Para la caliza, con un tiempo de precalentamiento 60 minutos, un tamaño de partícula $1 \mathrm{~cm}$ y un caudal de aire $0.0152 \mathrm{~m}^{3} / \mathrm{s}$ y empleando la ecuación 6 , se alcanza una temperatura promedio de $253.56^{\circ} \mathrm{C}$ y una recuperación de calor de $116.9 \mathrm{kcal}$, si lo confrontamos con una recuperación teórica del 100\% (266.49 kcal) la recuperación real es del $43.38 \%$. Para el coque, con un tiempo de precalentamiento 60 minutos, un tamaño de partícula $1 \mathrm{~cm}$ y un caudal de aire $0.0079 \mathrm{~m}^{3} / \mathrm{s}$ y empleando la ecuación 5, se alcanza una temperatura promedio de $302.68^{\circ} \mathrm{C}$ y una recuperación de calor de $37.2 \mathrm{kcal}$, si lo confrontamos con una recuperación teórica del 100\% (69.81 kcal) la recuperación real es del $53.32 \%$. 


\section{CONCLUSIONES}

La combinación de las variables adecuadas en el diseño de un recuperador de calor residual son fundamentales para un rendimiento óptimo, una relación $\mathrm{dT} / \mathrm{dp}$ de 6.4 , un tamaño de partícula de $1.5 \mathrm{~cm}$, un caudal de aire de $0.0224 \mathrm{~m}^{3} / \mathrm{s}$, en un tiempo de calentamiento de 60 minutos para la caliza, permiten obtener una temperatura promedio de $350{ }^{\circ} \mathrm{C}$, para el coque con una relación dT/dp de 6.4, con tamaño de partícula de $1.5 \mathrm{~cm}$ y con un caudal de $0.0079 \mathrm{~m}^{3} / \mathrm{s}$ en 60 minutos la temperatura promedio es superior a los $302^{\circ} \mathrm{C}$ sin entrar en etapa de ignición, lo que permite determinar que la recuperación de calor para la caliza es del $62 \%$ y para el coque es del orden de $53 \%$, Un recuperador como el propuesto en esta investigación puede permitir un mayor aprovechamiento energético al reutilizar el calor residual del horno vertical, disminuyendo los tiempos de calcinación de la caliza y el consumo de combustible, el medio ambiente se ve favorecido ya que se reduce ostensiblemente la contaminación térmica en planta, disminuyendo la temperatura de los gases de combustión, actúa como filtro del material particulado y de los gases de efecto invernadero, absorbiendo parte del monóxido y bióxido de carbono.

\section{RECOMENDACIONES}

El aire calentado a $546{ }^{\circ} \mathrm{C}$ da una buena aproximación de los efectos del precalentamiento, pero el contexto es que no es el gas real. Realizar un prototipo en planta permitirá determinar cuál es el calor recuperado real, cual es el efecto del recuperador sobre los gases de combustión y el material particulado y cuanto se puede reducir finalmente la contaminación térmica.

\section{REFERENCIAS}

[1].Sigma Thermal (2013 aprox.). Sistemas de calentamiento térmico. [Online]. Disponible en: http://www.sigmathermal.com/es/recuperacion-de-calorresidual.

[2] Zheng, Y.; Jie, Z.; Hong, C.(2005). Numerical simulation of thermal process and energy saving of lime furnace .J. Cent. South Univ. Technol, Vol. 12 No. 3. Jun. 1/205. Changsha 410083, China. Article ID. 1005- 9784(1/205)03 - 0295 - 05.

[3] Álvarez, F.J. y Oquendo,A.R. 1996.Montaje de una Planta Piloto para el Control de Emisiones en Horno de Cubilote. Trabajo de Grado - Pregrado en Ingeniería Metalúrgica, Universidad de Antioquia. 242 p.

[4] Mejía, R., Rivero, P.J. y Mejía, H.D. 2000.Propuesta de Número Adimensional para Optimización de Procesos
Termomecánicos. Revista Facultad de Ingeniería, Universidad de Antioquia. 20: 48-54.

[5] Mejía, R., Rivero, P.J. y Mejía, H.D.2001.Aproximación a la Función de Distribución de Temperatura de un Regenerador Térmico de Lecho Empacado. Revista Facultad de Ingeniería, Universidad de Antioquia. 23: 91-102.

[6] Mejía, R., Rivero, P.J. y Mejía, H.D.2002.Aproximación a la Función de Distribución de Temperatura de un Regenerador Térmico de Lecho Empacado, segunda parte. Revista Facultad de Ingeniería, Universidad de Antioquia. 25: 60-69.

[7] Giraldo, E. (2013). Desarrollo de un recuperador de calor de lecho empacado de caliza para el proceso de calcinación. Tesis de grado para obtener título de Magister. Instituto Metropolitano de Medellín.

[8] Benyahia, F. and Oneill, K. E. (2005). Enhanced voidage correlation for packed beds of various particle shapes and sizes. Particulate science and technology, 23. Taylor and Francis Inc. P. 169-167. 